\title{
STOCHASTIC PARABOLIC EQUATIONS OF FULL SECOND ORDER
}

\author{
S. V. LOTOTSKY AND B. L. ROZOVSKII
}

\begin{abstract}
A procedure is described for defining a generalized solution for stochastic differential equations using the Cameron-Martin version of the Wiener Chaos expansion. Existence and uniqueness of this Wiener Chaos solution is established for parabolic stochastic PDEs such that both the drift and the diffusion operators are of the second order.
\end{abstract}

\section{INTRODUCTION}

Consider a stochastic evolution equation

$$
d u(t)=(\mathcal{A} u(t)+f(t)) d t+(\mathcal{M} u(t)+g(t)) d W(t),
$$

where $\mathcal{A}$ and $\mathcal{M}$ are differential operators, and $W$ is a Wiener process on a probability space $(\Omega, \mathcal{F}, \mathbb{P})$. Traditionally, this equation was studied under the following assumptions:

- A1. The operator $\mathcal{A}$ is elliptic, the order of the operator $\mathcal{M}$ is less than the order of $\mathcal{A}$, and $\mathcal{A}-\frac{1}{2} \mathcal{M} \mathcal{M}^{\star}$ is elliptic (possibly degenerate) operator,

In fact, it is well known that unless assumption A1 holds, equation (1.1) has no solutions in $L_{2}(\Omega ; X)$ for any reasonable choice of the state space $X$.

It was shown recently (see [4, 5, 6] and the references therein) that if only the operator $\mathcal{A}$ is elliptic and the order of $\mathcal{M}$ is smaller than the order of $A$, then there exists a unique generalized solution of equation (1.1). This solution is often referred to as Wiener Chaos solution. It is given by the Wiener chaos expansion $u(t)=\sum_{|\alpha|<\infty} u_{\alpha}(t) \xi_{\alpha}$, where $\left\{\xi_{\alpha}\right\}_{|\alpha|<\infty}$ is the Cameron-Martin orthonormal basis in the space $L_{2}\left(\Omega ; \mathcal{F}^{W} ; X\right)$ of square integrable random elements in $X$ measurable with respect to to the sigma-algebra $\mathcal{F}^{W}$ generated by the Wiener process. The Cameron-Martin basis $\left\{\xi_{\alpha}\right\}$ is indexed by multiindices $\alpha=\left(\alpha_{1}, \alpha_{2}, \ldots\right)$. It was shown that for certain positive weights $Q=\{q(\alpha)\}_{|\alpha|<\infty}$, the weighted norm

$$
\|u\|_{Q, X}^{2}:=\sum_{|\alpha|<\infty} q^{2}(\alpha)\left\|u_{\alpha}\right\|_{L_{2}((0, T) ; X)}^{2}<\infty,
$$

S. V. Lototsky acknowledges support from NSF CAREER award DMS-0237724. B. L. Rozovskii acknowledges support from NSF Grant DMS 0604863, ARO Grant W911NF-07-1-0044, and ONR Grant N00014-07-1-0044. 
where $X$ is the appropriate Hilbert space characterizing the "regularity" of the solution. Note that without assumption A1

$$
E\|u\|_{L_{2}((0, T) ; X)}^{2}=\sum_{|\alpha|<\infty}\left\|u_{\alpha}(t)\right\|_{L_{2}((0, T) ; X)}^{2}=\infty .
$$

In this paper, we consider the Cauchy problem for the following stochastic partial differential equation:

$d u=\left(a_{i j} D_{i} D_{j} u+b_{i} D_{i} u+c u+f\right) d t+\left(\rho_{i j} D_{i} D_{j} u+\sigma_{i} D_{i} u+\nu u+g\right) d W, t \in[0, T], x \in \mathbb{R}$.

In contrast to the previous work, this is a parabolic SPDE of the full second order, in that the drift and diffusion operators have the same order 2. We construct a scale of weighted Wiener chaos spaces (related but not identical to Kondratiev's spaces) and prove the existence and uniqueness of the solution in the spaces from this scale.

\section{Constructing a Solution: an Example}

Let $\mathbb{F}=\left(\Omega, \mathcal{F},\left\{\mathcal{F}_{t}\right\}_{0<t<T}, \mathbb{P}\right)$ be a stochastic basis with the usual assumptions and $W=W(t), 0 \leq t \leq T$, a standard Wiener process on $\mathbb{F}$. For a Hilbert space $X$, denote by $L_{2}(\mathbb{W} ; X)$ the collection of $X$-valued random elements that are square integrable $\left(\mathbb{E}\|\cdot\|_{X}^{2}<\infty\right)$ and are measurable with respect to the sigma-algebra generated by $W(t), t \in[0, T]$.

Consider the Itô equation

$$
u(t, x)=e^{-x^{2} / 2}+\int_{0}^{t} u_{x x}(s, x) d s+\int_{0}^{t} u_{x x}(s, x) d W(s), t \in[0, T], x \in \mathbb{R} .
$$

If there is a solution, its Fourier transform in space, $\widehat{u}(t, y)=(1 / \sqrt{2 \pi}) \int_{\mathbb{R}} e^{-i x y} u(t, x) d x$ satisfies

$$
\widehat{u}(t, y)=e^{-y^{2} / 2}-y^{2} \int_{0}^{t} \widehat{u}(s, y) d s-y^{2} \int_{0}^{t} \widehat{u}(s, y) d W(t), t \in[0, T], y \in \mathbb{R} .
$$

For each fixed $y,(2.2)$ defines a geometric Brownian motion:

$$
\widehat{u}(t, y)=e^{-(1+t) y^{2}-\left(y^{4} / 2\right) t-y^{2} W(t)} .
$$

Let $H^{\gamma}(\mathbb{R})$ be the Sobolev space

$$
\left\{f: \int_{\mathbb{R}}\left(1+|y|^{2}\right)^{\gamma}|\widehat{f}(y)|^{2} d y<\infty\right\} .
$$

Since

$$
\mathbb{E}|\widehat{u}(t, y)|^{2}=e^{-2(1+t) y^{2}+y^{4} t},
$$

the solution of (2.1) cannot be an element of $L_{2}\left(\mathbb{W} ; L_{2}\left((0, T) ; H^{\gamma}(\mathbb{R})\right)\right)$ for any $\gamma \in \mathbb{R}$, even though the initial condition is non-random and is an element of $H^{\gamma}(\mathbb{R})$ for every $\gamma \in \mathbb{R}$. 
Let us try another approach. Once again, assuming that the solution exists, we apply the Itô formula to the product $u(t, x) \mathcal{E}_{h}(t)$, where

$$
\mathcal{E}_{h}(t)=\exp \left(\int_{0}^{t} h(s) d W(s)-\frac{1}{2} \int_{0}^{t} h^{2}(s) d s\right)
$$

and $h=h(t)$ is a smooth deterministic function. Since

$$
\mathcal{E}_{h}(t)=1+\int_{0}^{t} \mathcal{E}_{h}(s) h(s) d W(s),
$$

we conclude that the function

$$
u_{h}(t, x)=\mathbb{E}\left(u(t, x) \mathcal{E}_{h}(t)\right),
$$

if defined, must satisfy the heat equation

$$
u_{h}(t, x)=e^{-x^{2} / 2}+\int_{0}^{t}(1+h(s)) \frac{\partial^{2} u_{h}(s, x)}{\partial x^{2}} d s .
$$

If $\sup _{t}|h(t)|<1$, then this equation has a unique solution in every $H^{\gamma}(\mathbb{R})$ and

$$
u_{h}(t, x)=\mathbb{E} \exp \left(-(X(t, x))^{2} / 2\right),
$$

where

$$
X(t, x)=x+\int_{0}^{t} \sqrt{2(1+h(s))} d W(s) .
$$

In other words, while existence of a solution of equation (2.1) is still unclear, we now have a family of functions $u_{h}(t, x)$ defined by (2.10). All we need now is a systematic procedure of relating the family of deterministic functions $u_{h}=u_{h}(t, x)$ to a random process $u=u(t, x)$; then this process is natural to call a solution of (2.1).

Here is a possible way of constructing a stochastic process from $u_{h}$. Let $\mathfrak{m}=\left\{m_{k}, k \geq\right.$ $1\}$ be the Fourier cosine basis in $L_{2}((0, T))$ :

$$
m_{1}(s)=\frac{1}{\sqrt{T}} ; m_{k}(t)=\sqrt{\frac{2}{T}} \cos \left(\frac{\pi(k-1) t}{T}\right), k>1 ; 0 \leq t \leq T .
$$

Then

$$
h(t)=\sum_{k \geq 1} h_{k} m_{k}(t)
$$

For every fixed $t \in[0, T]$ and $\gamma \in \mathbb{R}$, we can now interpret the function $u_{h}(t, \cdot)$ as a mapping from the set of sequences $h=\left(h_{1}, h_{2}, \ldots\right)$ to the space $H^{\gamma}\left(\mathbb{R}^{d}\right)$, and, as equalities (2.10) and (2.11) suggest, this mapping is analytic in the region $\{h$ : $\left.\sum_{k>1} h_{k}^{2}<\varepsilon\right\}$ for sufficiently small $\varepsilon$. We will now compute the derivatives of this mapping.

Let $\mathcal{J}$ be the collection of multi-indices $\alpha=\left\{\alpha_{k}, k \geq 1\right\}$. Each $\alpha \in \mathcal{J}$ has nonnegative integer elements $\alpha_{k}$ and

$$
|\alpha|=\sum_{k} \alpha_{k}<\infty
$$


We also use the notation

$$
\alpha !=\prod_{k} \alpha_{k} !
$$

and consider special multi-indices, $\alpha=(0)$ with $|\alpha|=0$ and $\alpha=\varepsilon_{i}$, with $|\alpha|=1$, $\alpha_{i}=1$.

For each $\alpha \in \mathcal{J}$ define

$$
u_{\alpha}(t, x)=\left.\frac{1}{\sqrt{\alpha !}} \frac{\partial^{|\alpha|} u_{h}(t, x)}{\partial h_{1}^{\alpha_{1}} \partial h_{2}^{\alpha_{2}} \cdots}\right|_{h=0}
$$

Then

$$
u_{h}(t, x)=\sum_{\alpha \in \mathcal{J}} u_{\alpha}(t, x) \frac{h^{\alpha}}{\sqrt{\alpha !}}
$$

where

$$
h^{\alpha}=\prod_{k \geq 1} h_{k}^{\alpha_{k}}
$$

On the other hand, by direct computation,

$$
\mathcal{E}_{h}(t)=\mathbb{E}\left(\mathcal{E}_{h}(T) \mid \mathcal{F}_{t}^{W}\right)=\sum_{\alpha \in \mathcal{J}} \frac{h^{\alpha}}{\sqrt{\alpha !}} \xi_{\alpha}(t),
$$

where

$$
\xi_{\alpha}(t)=\mathbb{E}\left(\xi_{\alpha} \mid \mathcal{F}_{t}^{W}\right), \xi_{\alpha}=\frac{1}{\sqrt{\alpha !}} \prod_{k \geq 1} H_{\alpha_{k}}\left(\int_{0}^{T} m_{k}(t) d W(t)\right)
$$

and

$$
H_{n}(x)=(-1)^{n} e^{x^{2} / 2} \frac{d^{n}}{d x^{n}} e^{-x^{2} / 2}
$$

is $n$-th Hermite polynomial. It is a standard fact [1] that the collection $\left\{\xi_{\alpha}, \alpha \in \mathcal{J}\right\}$ is an orthonormal basis in $L_{2}(\mathbb{W} ; \mathbb{R})$.

The functions $u_{\alpha}(t, x), \alpha \in \mathcal{J}$, uniquely determine $u_{h}(t, x)$ according to (2.17). On the other hand, if

$$
\sum_{\alpha \in \mathcal{J}}\left\|u_{\alpha}(t)\right\|_{H^{\gamma}(\mathbb{R})}^{2}<\infty
$$

then the $H^{\gamma}(\mathbb{R})$-valued random process

$$
u(t, x)=\sum_{\alpha \in \mathcal{J}} u_{\alpha}(t, x) \xi_{\alpha}
$$

satisfies $\mathbb{E}\left(u(t, x) \mathcal{E}_{h}(t)\right)=u_{h}(t, x)$; if, in addition, $u$ is $\mathcal{F}_{t}^{W}$-adapted, then also

$$
u(t, x)=\sum_{\alpha \in \mathcal{J}} u_{\alpha}(t, x) \xi_{\alpha}(t) .
$$

If condition (2.22) fails, then (2.23) is a formal series, which we define to be the stochastic process corresponding to the family $u_{h}$. 
As (2.5) suggests, if $u_{h}$ is the solution of (2.9), then (2.22) fails for every $\gamma$. Let us now see how fast the series diverges. Equality (2.9) implies

$$
\begin{aligned}
u_{(0)}(t, x) & =e^{-x^{2} / 2}+\int_{0}^{t} \frac{\partial^{2} u_{(0)}(s, x)}{\partial x^{2}} d s,|\alpha|=0 \\
u_{\epsilon_{i}}(t) & =\int_{0}^{t} \frac{\partial^{2} u_{\epsilon_{i}}(s, x)}{\partial x^{2}} d s+\int_{0}^{t} \frac{\partial^{2} u_{(0)}(s, x)}{\partial x^{2}} m_{i}(s) d s,|\alpha|=1 \\
u_{\alpha}(t) & =\int_{0}^{t} \frac{\partial^{2} u_{\alpha}(s, x)}{\partial x^{2}} d s+\sum_{k=1}^{\infty} \sqrt{\alpha_{k}} \int_{0}^{t} \frac{\partial^{2} u_{\alpha-\epsilon_{k}}(s, x)}{\partial x^{2}} m_{k}(s) d s,|\alpha|>1 .
\end{aligned}
$$

Equations of the type (2.25) have been studied [4, Section 6 and References]. In particular, it is known that

$$
\sum_{|\alpha|=n}\left\|u_{\alpha}(t)\right\|_{H^{\gamma}(\mathbb{R})}^{2}=\frac{t^{n}}{n !}\left\|D_{x}^{2 n} \Phi_{t} u_{0}\right\|_{H^{\gamma}(\mathbb{R})}^{2},
$$

where $D_{x}=\partial / \partial x, \Phi_{t}$ is the heat semigroup, and $u_{0}(x)=e^{-x^{2} / 2}$. To simplify further computation, let us assume that $\gamma=0$. Then, switching to the Fourier transform,

$$
\left\|D_{x}^{2 n} \Phi_{t} u_{0}\right\|_{L_{2}\left(\mathbb{R}^{d}\right)}^{2}=\int_{\mathbb{R}}|y|^{4 n} e^{-y^{2}(t+1)} d y=\frac{\Gamma\left(2 n+\frac{1}{2}\right)}{(1+t)^{2 n} .}
$$

Using Stirling's formula for the Gamma function $\Gamma$,

$$
\sum_{|\alpha|=n}\left\|u_{\alpha}(t)\right\|_{L_{2}(\mathbb{R})}^{2}=\left(\frac{2 \sqrt{t}}{1+t}\right)^{2 n} C(n) n !
$$

where the numbers $C(n)$ are uniformly bounded from above and below. Similar result holds in every $H^{\gamma}(\mathbb{R})$. Thus, (2.22) does not hold, but instead, by (2.28), we have

$$
\sum_{\alpha \in \mathcal{J}} \frac{1}{|\alpha| !}\left\|u_{\alpha}(t)\right\|_{H^{\gamma}(\mathbb{R})}^{2}<\infty .
$$

We denote by $(\mathfrak{L})_{0,0}\left(\mathbb{W} ; H^{\gamma}(\mathbb{R})\right)$ the collection of formal series (2.24) satisfying (2.29); the reason for using $(\mathfrak{L})_{0,0}$ in the notation will become clear later. Note that we had equalities in all computations for equation (2.1) that lead to (2.29), which suggests that $(\mathfrak{L})_{0,0}\left(\mathbb{W} ; H^{\gamma}(\mathbb{R})\right)$ is the natural solution space for equation (2.1). For a more general stochastic parabolic equation of full second order in $\mathbb{R}^{d}$, the natural solution space turns out to be $(\mathfrak{L})_{p, q}\left(\mathbb{W} ; L_{2}\left((0, T) ; H^{\gamma}\left(\mathbb{R}^{d}\right)\right)\right)$ for suitable $p, q \leq 0$.

In the next section we address the following questions:

(1) How to define the spaces $(\mathfrak{L})_{p, q}(\mathbb{W} ; X)$ for $p, q \in \mathbb{R}$ without relying on an orthonormal basis in $L_{2}((0, T))$ ?

(2) How to construct a solution of a general stochastic parabolic equations of full second order? 


\section{General Constructions and the Main Result}

As before, let $\mathbb{F}=\left(\Omega, \mathcal{F},\left\{\mathcal{F}_{t}\right\}_{0 \leq t \leq T}, \mathbb{P}\right)$ be a stochastic basis with the usual assumptions and $W=W(t), 0 \leq t \leq T$, a standard Wiener process on $\mathbb{F}$. Denote by $H^{s}=H^{s}((0, T)), s \geq 0$, the Sobolev spaces on $(0, T)$ with norm

$$
\|\cdot\|_{s}=\left\|\Lambda^{s / 2} \cdot\right\|_{L_{2}((0, T))}
$$

where $\Lambda$ is the operator

$$
1-\frac{T^{2}}{\pi^{2}} \frac{d^{2}}{d t^{2}}
$$

with Neumann boundary conditions. This norm extends to functions of several variables via the tensor product of the spaces $H^{s}$.

Definition 3.1. Given real numbers $p, q$ and a Hilbert space $X,(\mathfrak{L})_{p, q}(\mathbb{W} ; X)$ is the closure of the set of $X$-valued random elements

$$
\eta=\eta_{0}+\sum_{k=1}^{N} \int_{0}^{T} \int_{0}^{s_{k}} \ldots \int_{0}^{s_{2}} \eta_{k}\left(s_{1}, \ldots, s_{k}\right) d W\left(s_{1}\right) \ldots d W\left(s_{k-1}\right) d W\left(s_{k}\right), \quad N \geq 1
$$

with respect to the norm

$$
\|\eta\|_{p, q ; X}^{2}=\left\|\eta_{0}\right\|_{X}^{2}+\sum_{k=1}^{N} \frac{2^{k p}}{(k !)^{2}}\|\| \eta_{k}\left\|_{q}\right\|_{X}^{2}
$$

where each $\eta_{k}, k \geq 1$, is a smooth symmetric function from $[0, T]^{k}$ to $X$.

Remark 3.2. (a) It is known [2, 7] that, for $\eta$ of the type (3.3),

$$
\mathbb{E}\|\eta\|_{X}^{2}=\left\|\eta_{0}\right\|_{X}^{2}+\sum_{k=1}^{N} \frac{1}{k !}\|\| \eta_{k}\left\|_{0}\right\|_{X}^{2}
$$

(b) The definition of each individual $(\mathfrak{L})_{p, q}(\mathbb{W} ; X)$ inevitably involves arbitrary choices, such as the norm in $H^{q}((0, T))$. Further analysis shows that different choices result in shifts of the indices $p, q$, and the space $\cup_{p, q}(\mathfrak{L})_{p, q}(\mathbb{W} ; X)$ does not depend on any arbitrary choices. In the white noise setting, where $\Omega$ is the space $\mathcal{S}^{\prime}\left(\mathbb{R}^{d}\right)$ of the Schwartz distributions and $\mathbb{P}$ is the normalized Gaussian measure on $\mathcal{S}$, the inductive limit $\cup_{p, q}(\mathfrak{L})_{p, q}(\mathbb{W} ; \mathbb{R})$ is the Kondratiev space $(\mathcal{S})_{-1}[2]$.

Remark 3.3. If $X$ is the Sobolev space $H^{\gamma}\left(\mathbb{R}^{d}\right)$, then we denote the norm $\|\cdot\|_{p, q ; X}$ by $\|\cdot\|_{p, q ; \gamma}$ :

$$
\|\cdot\|_{p, q ; H^{\gamma}\left(\mathbb{R}^{d}\right)}=\|\cdot\|_{p, q ; \gamma} .
$$

Proposition 3.4. Let $\eta=f \xi_{\alpha}$, where $f \in X$ and $\xi_{\alpha}$ is defined by (2.20). Then

$$
\|\eta\|_{p, q ; X}^{2}=\frac{2^{|\alpha| p}}{|\alpha| !} \mathbb{N}^{2 q \alpha}\|f\|_{X}^{2}
$$

where

$$
\mathbb{N}^{2 q \alpha}=\prod_{k \geq 1} k^{2 q \alpha_{k}}
$$


Proof. Let $|\alpha|=n$. It is known [3] that

$$
\xi_{\alpha}=\frac{1}{\sqrt{\alpha !}} \int_{0}^{T} \int_{0}^{s_{n}} \ldots \int_{0}^{s_{2}} E_{\alpha}\left(s_{1}, \ldots, s_{n}\right) d W\left(s_{1}\right) \ldots d W\left(s_{n-1}\right) d W\left(s_{n}\right),
$$

where $E_{\alpha}$ is the symmetric function

$$
E_{\alpha}\left(s_{1}, \ldots, s_{n}\right)=\sum_{\sigma \in \mathcal{P}_{n}} m_{i_{1}}\left(s_{\sigma(1)}\right) \ldots m_{i_{n}}\left(s_{\sigma(n)}\right) .
$$

In (3.10), the summation is over all permutations of $\{1, \ldots, n\}$, the functions $m_{k}$ are defined in (2.12), and the positive integer numbers $i_{1} \leq i_{2} \leq \ldots i_{n}$ are such that, for every sequence $\left(b_{k}, k \geq 1\right)$ of positive numbers,

$$
\prod_{k \geq 1} b_{k}^{\alpha_{k}}=b_{i_{1}} \cdot b_{i_{2}} \cdot \ldots \cdot b_{i_{n}} .
$$

For example, if $\alpha=(1,0,2,0,0,4,0,0, \ldots)$, then $|\alpha|=7$ and $i_{1}=1, i_{2}=i_{3}=3$, $i_{4}=\ldots=i_{7}=6$. Thus, in the notations of (3.4), we have

$$
\eta_{k}= \begin{cases}\frac{1}{\sqrt{\alpha !}} E_{\alpha} f, & \text { if } k=n \\ 0, & \text { otherwise }\end{cases}
$$

Note that

$$
\left\|E_{\alpha}\right\|_{0}=\sqrt{\alpha !} \sqrt{n !}
$$

By definition (3.2) of the operator $\Lambda$ we have

$$
\Lambda^{q / 2} m_{k}=k^{q} m_{k} \quad \text { or } \quad\left\|E_{\alpha}\right\|_{q}^{2}=\mathbb{N}^{2 q \alpha} \alpha ! n ! .
$$

The result now follows.

Corollary 3.5. A formal series

$$
\eta=\sum_{\alpha \in \mathcal{J}} \eta_{\alpha} \xi_{\alpha}
$$

with $\eta_{\alpha} \in X$, is an element of $(\mathfrak{L})_{p, q}(\mathbb{W} ; X)$ if and only if

$$
\sum_{\alpha \in \mathcal{J}} \frac{2^{p|\alpha|} \mathbb{N}^{2 q \alpha}}{|\alpha| !}\left\|\eta_{\alpha}\right\|_{X}^{2}<\infty
$$

Proof. This follows from (3.14) and the equality

$$
\left\|E_{\alpha}+E_{\beta}\right\|_{0}^{2}=\left\|E_{\alpha}\right\|_{0}^{2}+\left\|E_{\beta}\right\|_{0}^{2}, \alpha \neq \beta .
$$

Denote by $(\mathfrak{L})^{p, q}(\mathbb{W})$ the Hilbert space dual of $(\mathfrak{L})_{p, q}(\mathbb{W} ; \mathbb{R})$ relative to the inner product in $L_{2}(\mathbb{W} ; \mathbb{R})$, and by $\langle\langle\cdot, \cdot\rangle\rangle$ the corresponding duality. In the white noise setting, $\cap_{p, q}(\mathfrak{L})^{p, q}(\mathbb{W})$ is the space $(\mathcal{S})_{1}$ of the Kondratiev test functions [2]. If $\eta \in$ $(\mathfrak{L})_{p, q}(\mathbb{W} ; X)$ and $\zeta \in(\mathfrak{L})^{p, q}(\mathbb{W})$, then $\langle\langle\eta, \zeta\rangle\rangle$ is defined and belongs to $X$. 
For $h \in L_{2}((0, T))$, define

$$
\mathcal{E}_{h}=\mathcal{E}_{h}(T)=\exp \left(\int_{0}^{T} h(s) d W(s)-\frac{1}{2} \int_{0}^{T}|h(s)|^{2} d s\right) .
$$

Proposition 3.6. The random variable $\mathcal{E}_{h}$ is an element of $(\mathfrak{L})^{p, q}(\mathbb{W})$ if an only if

$$
\|h\|_{-q}^{2}<2^{p}
$$

Proof. Since

$$
\mathcal{E}_{h}(T)=1+\int_{0}^{T} h(t) \mathcal{E}_{h}(t) d t
$$

it follows that

$$
\mathcal{E}_{h}=1+\sum_{k=1}^{\infty} \int_{0}^{T} \int_{0}^{s_{k}} \cdots \int_{0}^{s_{2}} h\left(s_{k}\right) \cdots h\left(s_{1}\right) d W\left(s_{1}\right) \cdots d W\left(s_{k-1}\right) d W\left(s_{k}\right) .
$$

By (3.4) and (3.5), $\mathcal{E}_{h} \in(\mathfrak{L})^{p, q}(\mathbb{W})$ if and only if

$$
\sum_{k=1}^{\infty}\left(2^{-p}\|h\|_{-q}^{2}\right)^{k}<\infty
$$

that is, $\|h\|_{-q}^{2}<2^{p}$.

Definition 3.7. We say that the function $h$ is sufficiently small if (3.19) holds for sufficiently large (positive) $-p,-q$.

Proposition 3.8. If $u \in \bigcup_{p, q}(\mathfrak{L})_{p, q}(\mathbb{W} ; X)$ and $h$ is sufficiently small, then

$$
u_{h}=\left\langle\left\langle u, \mathcal{E}_{h}\right\rangle\right\rangle
$$

is an $X$-valued analytic function of $h$.

Proof. For every $u \in \bigcup_{p, q}(\mathfrak{L})_{p, q}(\mathbb{W} ; X)$, there exist $p, q$ such that $u \in(\mathfrak{L})_{p, q}(\mathbb{W} ; X)$; by Proposition 3.6, $u_{h}$ will indeed be defined for sufficiently small $h$. Similar to (2.17) we have

$$
u_{h}=\sum_{\alpha \in \mathcal{J}} \frac{u_{\alpha} h^{\alpha}}{\sqrt{\alpha !}}
$$

and this power series in $h^{\alpha}$ converges in some (infinite-dimensional) neighborhood of zero.

From now on, $D_{i}=\partial / \partial x_{i}$, and the summation convention is in force: $c_{i} d_{i}=\sum_{i} c_{i} d_{i}$, etc.

Consider the linear equation in $\mathbb{R}^{d}$

$$
d u=\left(a_{i j} D_{i} D_{j} u+b_{i} D_{i} u+c u+f\right) d t+\left(\rho_{i j} D_{i} D_{j} u+\sigma_{i} D_{i} u+\nu u+g\right) d W
$$

with initial condition $u(0, x)=v(x)$, under the following assumptions:

B0 All coefficients are non-random. 
B1 The functions $a_{i j}=a_{i j}(t, x), \rho_{i j}=\rho_{i j}(t, x)$ are measurable and bounded in $(t, x)$ by a positive number $C_{0}$, and

(i)

$$
\left|a_{i j}(t, x)-a_{i j}(t, y)\right|+\left|\rho_{i j}(t, x)-\rho_{i j}(t, y)\right| \leq C_{0}|x-y|, x, y \in \mathbb{R}^{d}, 0 \leq t \leq T
$$

(ii) the matrix $\left(a_{i j}\right)$ is uniformly positive definite, that is, there exists a $\delta>0$ so that, for all vectors $y \in \mathbb{R}^{d}$ and all $(t, x), a_{i j} y_{i} y_{j} \geq \delta|y|^{2}$.

B2 The functions $b_{i}=b_{i}(t, x), c=c(t, x), \sigma_{i}=\sigma_{i}(t, x)$, and $\nu=\nu(t, x)$ are measurable and bounded in $(t, x)$ by the number $C_{0}$.

B2

$$
u_{0} \in \bigcup_{p, q}(\mathfrak{L})_{p, q}\left(\mathbb{W} ; L_{2}\left(\mathbb{R}^{d}\right)\right), f, g \in \bigcup_{p, q}(\mathfrak{L})_{p, q}\left(\mathbb{W} ; L_{2}\left((0, T) ; H^{-1}\left(\mathbb{R}^{d}\right)\right)\right)
$$

For simplicity, we introduce the following notations for the differential operators in (3.25):

$$
\mathcal{A}=a_{i j} D_{i} D_{j}+b_{i} D_{i}+c, \quad \mathcal{B}=\rho_{i j} D_{i} D_{j}+\sigma_{i} D_{i}+\nu .
$$

Definition 3.9. A solution $u$ of $(3.25)$ is an element of $\bigcup_{p, q}(\mathfrak{L})_{p, q}\left(\mathbb{W} ; L_{2}\left((0, T) ; H^{1}\left(\mathbb{R}^{d}\right)\right)\right)$ such that, for all sufficiently small $h$ and all $t \in[0, T]$, the equality

$$
u_{h}(t, x)=v_{h}(x)+\int_{0}^{t}(\mathcal{A}+h(s) \mathcal{B}) u_{h}(s, x) d s
$$

holds in $H^{-1}\left(\mathbb{R}^{d}\right)$.

The following theorem is the main result of this paper.

Theorem 3.10. Assume that, for some $p>0$ and $q>1, u_{0} \in(\mathfrak{L})_{p, q}\left(\mathbb{W} ; L_{2}\left(\mathbb{R}^{d}\right)\right)$ and $f, g$ are elements of the space $(\mathfrak{L})_{p, q}\left(\mathbb{W} ; L_{2}\left((0, T) ; H^{-1}\left(\mathbb{R}^{d}\right)\right)\right)$. Then there exist $r, \ell<0$ such that equation (3.25) has a unique solution $u \in(\mathfrak{L})_{r, \ell}\left(\mathbb{W} ; L_{2}\left((0, T) ; H^{1}\left(\mathbb{R}^{d}\right)\right)\right)$ and

$$
\int_{0}^{T}\|u(t)\|_{r, \ell ; 1}^{2} d t \leq C \cdot\left(\|v\|_{p, q ; 0}^{2}+\int_{0}^{T}\left(\|f(t)\|_{p, q ;-1}^{2}+\|g(t)\|_{p, q ;-1}^{2}\right) d t\right) .
$$

The number $C>0$ depends only on $\delta, C_{0}, p, q, r, \ell$, and $T$.

Proof. The proof consists of two steps: first, we prove the result for deterministic functions $v, f, g$ and then use linearity to extend the result to the general case.

Step 1. Assume that the functions $v \in L_{2}\left(\mathbb{R}^{d}\right), f, g \in L_{2}\left((0, T) ; H^{-1}\left(\mathbb{R}^{d}\right)\right)$ are deterministic. Then $v_{h}=v, f_{h}=f, g_{h}=g$, and classical theory of parabolic equations shows that, for sufficiently small $h$, equation (3.28) has a unique solution $u_{h}$ and the dependence of $u_{h}$ on $h$ is analytic.

As in the previous section, we write

$$
u(t, x)=\sum_{\alpha \in \mathcal{J}} u_{\alpha}(t, x) \xi_{\alpha}
$$


where the coefficients $u_{\alpha}$ satisfy

$$
\begin{aligned}
u_{(0)}(t, x) & =v(x)+\int_{0}^{t}\left(\mathcal{A} u_{(0)}(s, x)+f(s, x)\right) d s \\
u_{\epsilon_{k}}(t, x) & =\int_{0}^{t} \mathcal{A} u_{\epsilon_{k}}(s, x) d s+\int_{0}^{t}\left(\mathcal{B} u_{(0)}(s, x)+g(s, x)\right) m_{k}(s) d s \\
u_{\alpha}(s, x) & =\int_{0}^{t} \mathcal{A} u_{\alpha}(s, x) d s+\sum_{k} \sqrt{\alpha_{k}} \int_{0}^{t} \mathcal{B} u_{\alpha-\epsilon_{k}}(s, x) m_{k}(s) d s,|\alpha|>1 .
\end{aligned}
$$

Denote by $\Phi=\Phi_{s, t}, t \geq s \geq 0$ the semigroup generated by the operator $\mathcal{A}$. It follows by induction on $|\alpha|$ that

$$
\begin{aligned}
& u_{(0)}(t, x)=\Phi_{t, 0} v(x)+\int_{0}^{t} \Phi_{t, s} f(s) d s \\
& u_{\epsilon_{k}}(t, x)=\int_{0}^{t} \Phi_{t, s}\left(\mathcal{B} u_{(0)}(s, x)+g(s, x)\right) m_{k}(s) d s \\
& u_{\alpha}(t, x)=\frac{1}{\sqrt{\alpha !}} \int_{0}^{t} \int_{0}^{s_{n}} \ldots \int_{0}^{s_{2}} \Phi_{t, s_{n}} \mathcal{B} \Phi_{s_{n}, s_{n-1}} \cdots \mathcal{B} \Phi_{s_{2}, s_{1}}\left(\mathcal{B} u_{(0)}\left(s_{1}, x\right)+g\left(s_{1}, x\right)\right) \\
& E_{\alpha}\left(s_{1}, \ldots, s_{n}\right) d s_{1} \ldots d s_{n},|\alpha|=n>1 .
\end{aligned}
$$

Therefore, using the usual parabolic estimates,

$$
\int_{0}^{T}\left\|u_{\alpha}(t)\right\|_{H^{1}\left(\mathbb{R}^{d}\right)}^{2} d t \leq \frac{C^{n} n !}{\alpha !}\left(\|v\|_{L_{2}\left(\mathbb{R}^{d}\right)}^{2}+\int_{0}^{T}\left(\|f(t)\|_{H^{-1}\left(\mathbb{R}^{d}\right)}^{2}+\|g(t)\|_{H^{-1}\left(\mathbb{R}^{d}\right)}^{2}\right) d t\right)
$$

and then (3.29) follows from (3.16).

Step 2. As in Step 1, existence and uniqueness of solution follows from unique solvability of the parabolic equation (3.28), and it remains to establish (3.29).

Denote by $u(t, x ; V, F, G, \gamma), \gamma \in \mathcal{J}$, the solution of (3.25) with $v=V \xi_{\gamma}, f=F \xi_{\gamma}$, $g=G \xi_{\gamma}$. If $v=\sum_{\alpha \in \mathcal{J}} v_{\alpha} \xi_{\alpha}$, etc., then

$$
u(t, x)=\sum_{\gamma \in \mathcal{J}} u\left(t, x ; v_{\gamma}, f_{\gamma}, g_{\gamma}, \gamma\right)
$$

It follows from (3.31) that $u_{\alpha}(t, x ; V, F, G, \gamma)=0$ if $|\alpha|<|\gamma|$ and

$$
\frac{u_{\alpha+\gamma}(t, x ; V, F, G, \gamma)}{\sqrt{(\alpha+\gamma) !}}=\frac{u_{\alpha}\left(t, x ; \frac{V}{\sqrt{\gamma !}}, \frac{F}{\sqrt{\gamma !}}, \frac{G}{\sqrt{\gamma !}},(0)\right)}{\sqrt{\alpha !}} .
$$

Using the results of Step 1,

$$
\begin{aligned}
& \int_{0}^{T}\left\|u\left(t, \cdot ; v_{\gamma}, f_{\gamma}, g_{\gamma}\right)\right\|_{r, \ell ; 1}^{2} d t \\
& \quad \leq \frac{C}{\gamma !}\left(\left\|v_{\gamma}\right\|_{L_{2}\left(\mathbb{R}^{d}\right)}^{2}+\int_{0}^{T}\left(\left\|f_{\gamma}(t)\right\|_{H^{-1}\left(\mathbb{R}^{d}\right)}^{2}+\left\|g_{\gamma}(t)\right\|_{H^{-1}\left(\mathbb{R}^{d}\right)}^{2}\right) d t\right) .
\end{aligned}
$$

Now (3.29) follows from (3.34) by the triangle inequality. 


\section{REFERENCES}

[1] R. H. Cameron and W. T. Martin. The orthogonal development of nonlinear functionals in a series of Fourier-Hermite functions. Ann. Math., 48(2):385-392, 1947.

[2] H. Holden, B. Øksendal, J. Ubøe, and T. Zhang. Stochastic Partial Differential Equations. Birkhäuser, Boston, 1996.

[3] K. Ito. Multiple Wiener integral. J. Math. Soc. Japan, 3:157-169, 1951.

[4] S. V. Lototsky and B. L. Rozovskii. Stochastic differential equations: a Wiener chaos approach. In Yu. Kabanov, R. Liptser, and J. Stoyanov, editors, From stochastic calculus to mathematical finance: the Shiryaev festschrift, pages 433-507. Springer, 2006.

[5] S. V. Lototsky and B. L. Rozovskii. Wiener chaos solutions of linear stochastic evolution equations. Ann. Probab., 34(2):638-662, 2006.

[6] R. Mikulevicius and B. L. Rozovskii. Linear parabolic stochastic PDE's and Wiener Chaos. SIAM J. Math. Anal., 292:452-480, 1998.

[7] D. Nualart. Malliavin Calculus and Related Topics, 2nd Edition. Springer, New York, 2006.

Current address, S. V. Lototsky: Department of Mathematics, USC, Los Angeles, CA 90089

E-mail address, S. V. Lototsky: lototsky@math.usc.edu

$U R L:$ http://www-rcf.usc.edu/ lototsky

Current address, B. L. Rozovskii: Division of Applied Mathematics, Brown University, Providence, RI 02912

E-mail address, B. L. Rozovskii: rozovsky@dam.brown.edu 